\title{
Thermal effects in InP/(Ga,In)P quantum-dot single-photon emitters
}

\author{
A. K. Nowak, ${ }^{1}$ E. Gallardo, ${ }^{1}$ D. Sarkar, ${ }^{1,2}$ H. P. van der Meulen, ${ }^{1}$ J. M. Calleja, ${ }^{1}$ J. M. Ripalda,${ }^{3}$ L. González, ${ }^{3}$ and \\ Y. González ${ }^{3}$ \\ ${ }^{1}$ Departamento de Física de Materiales, Universidad Autónoma de Madrid, E-28049 Madrid, Spain \\ ${ }^{2}$ Department of Physics and Astronomy, University of Sheffield, Sheffield S3 7RH, United Kingdom \\ ${ }^{3}$ Instituto de Microelectrónica de Madrid, Centro Nacional de Microelectrónica, Consejo Superior de Investigaciones Científicas, \\ Isaac Newton 8, PTM Tres Cantos, E-28760 Madrid, Spain
}

(Received 14 July 2009; revised manuscript received 29 September 2009; published 14 October 2009)

\begin{abstract}
We present second-order photon correlation measurements on single $\mathrm{InP} /(\mathrm{Ga}, \mathrm{In}) \mathrm{P}$ quantum dots as a function of temperature. Low background emission allows to obtain antibunching minima $g^{(2)}(0)$ below 0.25 up to $45 \mathrm{~K}$. The antibunching time $\tau_{R}$ increases or decreases with temperature depending on the quantum-dot size. The two trends result from a competition between hole thermal excitation and dark-to-bright exciton transitions. The former prevails in smaller dots showing increasing $\tau_{R}$ with temperature, while the latter dominates in larger quantum dots showing decreasing $\tau_{R}$ with temperature.
\end{abstract}

DOI: 10.1103/PhysRevB.80.161305

PACS number(s): 78.67.Hc, 78.55.Cr, 42.50.Ar

Semiconductor quantum dots (QDs) are among the most promising single-photon emitters (SPEs) for quantum information applications due to their versatility, scalability, and ease to handle as compared to atom or ion-based SPEs. ${ }^{1-4}$ However, the use of semiconductor QDs as true "on demand" SPEs is conditioned by the presence of "background photons" (photons emitted outside the QD but at the QD energy) and decoherence. ${ }^{5}$ One important source of decoherence in QDs is the random transition between bright exciton (BX) and dark exciton (DX) states (exciton states with total angular momentum 1 and 2, respectively). ${ }^{6}$ Exciton energy splittings, as the dark-bright exciton splitting $E_{\mathrm{DB}}$ and the fine-structure splitting $\Delta_{\mathrm{FS}}$, which are large in QDs as compared to higher dimensional systems due to the increased electron-hole Coulomb interaction, are strongly sensitive to the QD size and shape. ${ }^{7-9}$ InP QDs have received special attention for their potential use as SPEs in the visible range. ${ }^{10-16}$ Photon correlation measurements for both continuous $^{9,11}$ and pulsed excitation ${ }^{11,13,15}$ as well as under electrical injection ${ }^{14}$ show clear antibunching dips in the second-order photon correlation function $g^{(2)}(\tau)$ at zero delay $(\tau=0)$. The standard form of the correlation function is

$$
g^{(2)}(\tau)=1-\beta \exp \left(-|\tau| / \tau_{R}\right),
$$

where $\tau_{R}$ is the characteristic (minimum) time needed for the emission of a second photon after the first one has been emitted by the QD and $\beta$ is generally determined by background photons. A value $\beta=1$ indicates perfect SPE. The low values of $g^{(2)}(0)$ found in InP QDs (between 0.1 and 0.2 ) (Refs. 10-15) is indicative of efficient single-photon emission. High-temperature operation of a SPE is beneficial for practical uses. Antibunching dips up to $200 \mathrm{~K}$ have been reported for GaN (Ref. 17) and CdSe (Ref. 18) QDs and up to $90 \mathrm{~K}$ in InGaAs/AlGaAs QDs. ${ }^{19}$ In InP QDs an upper limit of $80 \mathrm{~K}$ has been reached using $\mathrm{Al}$ containing barriers. ${ }^{13}$ Upon raising temperature the $g^{(2)}(0)$ value progressively increases due to the increasing background luminescence. Temperature also influences the antibunching width. Indeed $\tau_{R}$ depends on several factors, as the pumping rate, the exciton lifetime ${ }^{20}$ and the carrier relaxation time, some of them being temperature dependent. An increase in $\tau_{R}$ with temperature has been reported in InGaAs/AlGaAs QDs. ${ }^{19}$

In this Rapid Communication we present photon correlation measurements on small InP/GaInP QDs to study the effect of temperature on $g^{(2)}(0)$ and $\tau_{R}$. Single QDs are selected that emit at high energies, well outside the ensemble photoluminescence (PL) emission, to minimize the interdot contribution to the background emission. We obtain $g^{(2)}(0)$ values below 0.25 up to $45 \mathrm{~K}$, as a result of the low background. Increasing temperature produces either an increase or a decrease in $\tau_{R}$ depending on the QD size. The size is inferred from the relative values of the biexciton binding energy $E_{b}^{\mathrm{XX}}$ and the fine-structure splitting $\Delta_{\mathrm{FS}}{ }^{8}$ We propose a competition between the dark-to-bright exciton transition (governed by $E_{\mathrm{DB}}$ ) and thermal excitation of holes (governed by an effective interhole energy spacing $E_{\mathrm{h}}$ ) to explain the different behavior of $\tau_{R}$. Thermal hole excitation is dominant for smaller QDs in which $\tau_{R}$ increases with temperature, ${ }^{19}$ while dark-to-bright exciton transitions are favored in larger QDs, showing decreasing $\tau_{R}$.

The QD samples have been grown by molecular-beam epitaxy on GaAs (001) substrates. The growth sequence was $100 \mathrm{~nm}$ GaInP, 2 monolayers (ML) GaAs, and 2.2 ML InP, repeated twice. The critical thickness for QD nucleation at $470{ }^{\circ} \mathrm{C}$ was $2 \mathrm{ML}$ of $\mathrm{InP}$ at a growth rate $0.05 \mathrm{ML} / \mathrm{s}$. The second uncapped layer of QDs was used for atomic force microscopy characterization. The QD average diameter and height before capping are 35 and $6 \mathrm{~nm}$, respectively. However, the single QD selected for this work are far in the high energy tail of the ensemble PL so that their height is much less than $6 \mathrm{~nm}$. From their PL energies we estimate it to be between 1 and $2 \mathrm{~nm} .{ }^{15} \mathrm{PL}$ spectra of QDs were taken through a $100 \times$ microscope objective $(1.5 \mu \mathrm{m}$ spot size) under nonresonant excitation using a $\mathrm{He}-\mathrm{Ne}$ laser and a charge coupled device detector located at one of the exits of a $0.75 \mathrm{~cm}$ focal length spectrometer. The photon correlation measurements were done with a Hanbury-Brown and Twiss (HBT) interferometer located at the second exit of the spectrometer. Two avalanche photodiodes with $65 \%$ efficiency at the QD emission energy $(1.86 \mathrm{eV})$ were used for coincidence detection. 


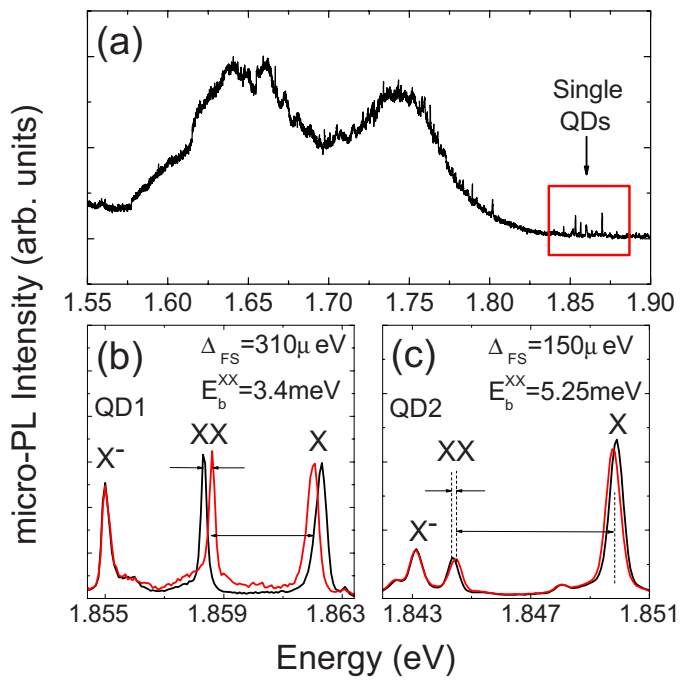

FIG. 1. (Color online) (a) PL spectrum of the InP/GaInP QD ensemble. (b) and (c) PL spectra of single quantum dots: QD1 and QD2, respectively. The black and red lines represent polarization parallel to the [110] and [1-10] crystallographic directions, respectively. The chosen quantum dots exhibit large differences in the binding energy $E_{b}^{\mathrm{XX}}$ and the fine-structure splitting $\Delta_{\mathrm{FS}}$ indicating different QD size and/or shape.

Their response time $\left(\tau_{\mathrm{i}}=0.5 \mathrm{~ns}\right)$ was measured with $2 \mathrm{ps}$ pulses of a Ti-sapphire laser. Count rates at the detectors were up to $10^{4}$ counts/s. The ensemble PL spectrum of the QDs is presented in Fig. 1(a). Single QDs were selected in the 1.84-1.87 eV emission range, well above the average emission energy. This ensures small QD size allows spectral isolation of single QD emission lines and prevents charge transfer from neighboring QDs. The micro-PL spectra of two different quantum dots are shown in Figs. 1(b) and 1(c) for relatively high excitation power. Three lines (exciton $X$, biexciton $X X$, and negatively charged exciton $X^{-}$) are identified by their intensity dependence on excitation power (not shown) and linear polarization. Black and red traces correspond to linear polarization parallel to the [110] and [1-10] crystallographic directions, respectively. Significant differences in the biexciton binding energy $\left(E_{b}^{\mathrm{XX}}\right)$ and the finestructure splitting are observed between QD1 and QD2. Both the smaller value of $E_{b}^{\mathrm{XX}}$ and the higher value of $\Delta_{\mathrm{FS}}$ indicate stronger electron-hole exchange energy ${ }^{8}$ in QD1. As exchange energy depends on the electron-hole wave-function overlap, we infer that QD1 is significantly smaller in height than QD2.

The second-order photon autocorrelation function of the exciton was measured for both quantum dots in the temperature range between 5 and $45 \mathrm{~K}$. The result for QD2 at $5 \mathrm{~K}$ is shown in Fig. 2. Solid and dashed lines are fits to Eq. (1) with $\left(g_{\text {CONV }}^{(2)}(\tau)\right)$ and without $\left(g^{(2)}(\tau)\right)$ convolution with the instrumental time response function, respectively, which is proportional to $\exp \left(-|\tau| / \tau_{i}\right) .{ }^{14}$ As the background PL intensity is only $2 \%$ of the QD emission, no background correction was done. ${ }^{21}$ The $g^{(2)}(0)$ and $\tau_{R}$ values corrected for the instrumental response time are $0.07 \pm 0.05$ and $0.63 \pm 0.16 \mathrm{~ns}$, respectively. Error bars resulting from fit to Eq. (1) are mainly due to the relatively high noise level in the HBT histogram.

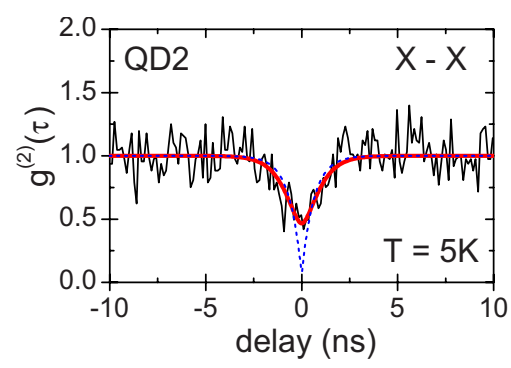

FIG. 2. (Color online) Second-order correlation function of $X$ emission from QD2. The solid and dashed lines are theoretical fits to Eq. (1) with $\left(g_{\text {CONV }}^{(2)}(\tau)\right)$ and without $\left(g^{(2)}(\tau)\right)$ convolution with the instrumental time response function, respectively. The strong antibunching minimum at zero time delay implies close to ideal single-photon emission from the QD.

The variations of $g^{(2)}(0)$ and $\tau_{R}$ with temperature constitute the main point of this Rapid Communication. We will discuss them together with the temperature dependence of the PL intensity ratio $I_{X} /\left(I_{X}+I_{\mathrm{XX}}\right)$, where $I_{X}$ and $I_{\mathrm{XX}}$ are the exciton and biexciton emission intensities, respectively, to get better insight into the involved mechanisms. The temperature dependence of $I_{X} /\left(I_{X}+I_{\mathrm{XX}}\right), \tau_{R}$ and $g_{\mathrm{CONV}}^{(2)}(0)$, are shown in Fig. 3 for QD1 [Figs. 3(a), 3(c), and 3(e)] and QD2 [Figs. 3(b), 3(d), and 3(f)], respectively. The data correspond to low excitation intensity so that $I_{\mathrm{XX}} \leq 0.15 I_{X}$. We observe marked differences between QD1 and QD2. The intensity ratio increases steadily with temperature for QD1 while it is almost constant up to $40 \mathrm{~K}$ for QD2 and increases at higher

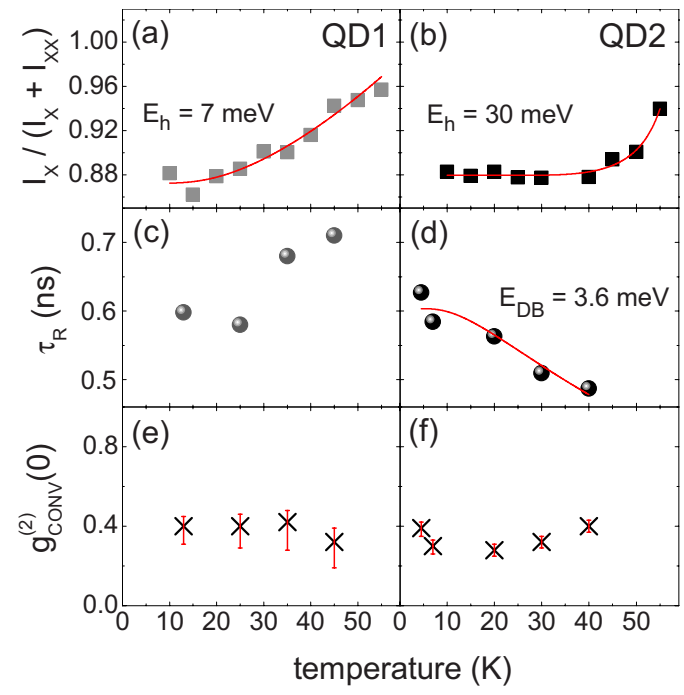

FIG. 3. (Color online) Temperature dependence of quantum-dot properties for QD1 (left) and QD2 (right): (a) and (b) PL intensity ratio $I_{X} /\left(I_{X}+I_{\mathrm{XX}}\right)$, where $I_{X}$ and $I_{\mathrm{XX}}$ are the exciton and biexciton intensities, respectively. The solid lines are fits to Eq. (3). (c) and (d) Characteristic antibunching time $\tau_{R}$. The solid line is the fit to Eq. (4). The differences between QD1 and QD2 observed both in the PL intensity ratio and in $\tau_{R}$ show competition between hole thermal excitation and dark-to-bright exciton transition processes. (e) and (f) Antibunching minima obtained from the experimental data before deconvolution with the instrumental time response function. 
temperatures. The antibunching time increases for QD1 and decreases for QD2, as shown in Figs. 3(c) and 3(d).

Among the possible mechanisms influencing $\tau_{R}$ (exciton lifetime, pump rate, etc. $)^{20}$ and $I_{X} /\left(I_{X}+I_{\mathrm{XX}}\right)$, there are at least two temperature dependent ones: dark-to-bright exciton transitions ${ }^{22,23}(D \rightarrow B)$ and thermal excitation of holes. ${ }^{19}$ The first one is expected to reduce $\tau_{R}$ as $D \rightarrow B$ events produce bright excitons in addition to those formed by direct capture of an electron-hole pair (EHP) with proper angular momenta by the QD. The second mechanism (hole excitation) increases $\tau_{R}$ as it reduces the probability of ground-state exciton formation. We discuss next the effect of both mechanisms on $I_{X} /\left(I_{X}+I_{\mathrm{XX}}\right)$ and $\tau_{R}$.

$D \rightarrow B$ transitions have been claimed as the origin of the $I_{X} /\left(I_{X}+I_{\mathrm{XX}}\right)$ increase with temperature, and $E_{\mathrm{DB}}$ values between 1.4 and $5 \mathrm{meV}$ have been reported in small InP QDs. ${ }^{15}$ Thermal hole excitation will also increase $I_{X} /\left(I_{X}+I_{X X}\right)$. It decreases the emission probability of both $X$ and $X X$, but while excitation of the only hole present in $X$ just reduces $I_{X}$, excitation of one of the two holes present in $X X$ reduces $I_{\mathrm{XX}}$ and leaves a new exciton. Thus $I_{X X}$ is expected to decrease faster than $I_{X}$ upon thermal excitation of holes. Which mechanism dominates will depend on the respective characteristic energies: $E_{\mathrm{DB}}$ for $D \rightarrow B$ and $E_{\mathrm{h}}$ for hole excitation. In our case, a data fit with an Arrhenius-type law involving $D \rightarrow B$ processes $^{15}$ gives activation energies of the order of tens of meV for QD1 and QD2. These values are inconsistent with any reasonable estimate of $E_{\mathrm{DB}}$ in InP QDs. ${ }^{15,24}$ Instead, they are close to the expected values of the excited hole states, so that thermal excitation of holes could account for the observed temperature trend in our case. A rough estimate of $E_{\mathrm{h}}$ can be obtained assuming that $I_{X}$ and $I_{\mathrm{XX}}$ decrease with temperature according to

$$
\begin{gathered}
I_{X}=A-B N, \\
I_{X X}=C-D N, \\
N=\left[\exp \left(E_{h} / k T\right)-1\right]^{-1},
\end{gathered}
$$

where $N$ is the Bose-Einstein occupation factor of the phonons responsible for the hole excitation, and $A, B, C$, and $D$ are temperature independent constants. Then we have

$$
\frac{I_{X}}{I_{X}+I_{X X}}=\frac{1-a N}{b-c N},
$$

where $a=B / A, c=(B+D) / A$, and $b=1+C / A$. The parameter $\mathrm{b}$ is fixed by the intensity ratio at low temperature. The fit of our $I_{X} /\left(I_{X}+I_{X X}\right)$ data with Eq. (3) [continuous lines in Figs. $3(\mathrm{a})$ and $3(\mathrm{~b})]$ gives $E_{\mathrm{h}}=7$ and $E_{\mathrm{h}}=30 \mathrm{meV}$ for QD1 and QD2, respectively. These values are compatible with the heavy-light hole splitting in our QDs, which show a weak hole confinement. Actually the valence-band offset of unstrained $\mathrm{InP} / \mathrm{InGaP}$ is negative $\left(\Delta E_{\mathrm{V}}=-45 \mathrm{meV}\right)$ (Ref. 25) so that holes are solely confined by strain. In our small QDs only one pair of confined hole states (heavy and light) is expected. For a 1-nm-high QD with rectangular confinement potential and positive valence-band offset of $50 \mathrm{meV}$, a heavy-light hole splitting of $E_{\mathrm{h}}=6 \mathrm{meV}$ is expected. This

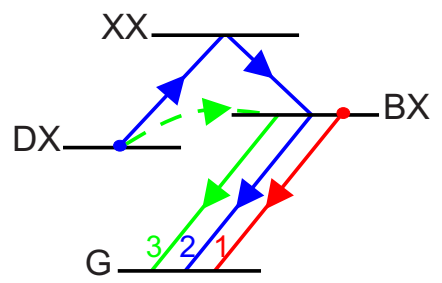

FIG. 4. (Color online) Level scheme including: QD ground state $(g)$, DX, BX, and biexciton $(X X)$. The arrows $(1,2$, and 3$)$ represent the photon emission of the bright exciton state after three possible ways of charging.

value increases upon increase of the QD height, i.e., moving from QD1 to QD2.

Going now to $\tau_{R}$ we recall that our measurements are done at low excitation intensities. In these conditions bright excitons recombine before capture of a second EHP to form a biexciton. Biexcitons are thus formed mainly from dark excitons, while bright excitons can result from three different channels (see Fig. 4): (1) capture by the empty QD of an EHP with antiparallel angular momenta directly from the wetting layer (red arrow), (2) emission of a biexciton photon (blue arrows), and (3) a thermally activated $D \rightarrow B$ exciton transition assisted by acoustic phonons. ${ }^{23}$ The two first channels are roughly temperature independent. Thus, the antibunching time $\tau_{R}$ can be represented by

$$
\begin{gathered}
\frac{1}{\tau_{R}}=\frac{1}{\tau_{A}}+\frac{1}{\tau_{B}}, \\
\frac{1}{\tau_{B}}=A\left[\exp \left(E_{\mathrm{DB}} / k T\right)-1\right]^{-1},
\end{gathered}
$$

where $1 / \tau_{A}$ is the combined transition probability for channels 1 and 2 , while $1 / \tau_{B}$ stands for channel 3 . Here we assume a $D \rightarrow B$ transition activated by acoustic phonons with energies close to $E_{\mathrm{DB}}$. The temperature trend of QD2 is properly described by Eq. (4) [solid line in Fig. 3(d)] giving $E_{\mathrm{DB}}=3.6 \mathrm{meV} . E_{\mathrm{DB}}$ values of this magnitude are not surprising in spite of the lower values found in InAs QDs. ${ }^{26,27}$ In fact, values of the order of a few $\mathrm{meV}$ have been reported both theoretically ${ }^{24}$ and experimentally ${ }^{15}$ in small InP QDs due to the increased electron-hole exchange interaction. This is also consistent with the high $E_{\mathrm{DB}}$ value $(2 \mathrm{meV})$ found in CdSe QDs with a fine-structure splitting (200 $\mu \mathrm{eV})$ (Ref. 28) comparable to the present ones $(150-300 \mu \mathrm{eV})$. In the smaller QD1 we expect a larger value of $E_{\mathrm{DB}}$ due to the increased electron-hole overlap and a lower $E_{\mathrm{h}}$ due to the increased confining energy. The increasing trend of $\tau_{R}$ with temperature indicates that thermal excitation of holes takes over $D \rightarrow B$ processes $\left(E_{\mathrm{h}} \leq E_{\mathrm{DB}}\right)$ in QD1. Indeed, if the probability of finding a hole in the ground state decreases, the average time needed to "recharge" the QD after emission of a bright exciton photon will increase. As a result $E_{\mathrm{h}}$ dominates at low $T$ and $\tau_{R}$ increases with temperature in QD1. In the larger QD2 it is the other way around. Smaller $E_{\mathrm{DB}}$ and larger $E_{\mathrm{h}}$ values lead to $E_{\mathrm{h}}>E_{\mathrm{DB}}$. In this case $E_{\mathrm{DB}}$ dominates over $E_{\mathrm{h}}$ at low temperature, leading to a $\tau_{R}$ decrease. 
As a final remark, we note that the $g_{\mathrm{CONV}}^{(2)}(0)$ values obtained from the raw experimental data reported in this work remain below 0.48 and 0.43 for QD1 and QD2, respectively, as shown in Figs. 3(e) and 3(f) for the whole temperature range. Deconvolution of the experimental data with the instrumental time response function returns average $g^{(2)}(0)$ values below 0.25 for both QDs, without the need of background subtraction. The low $g^{(2)}(0)$ values reported here open good perspectives to use these small InP QDs as efficient SPEs at moderate temperatures.

In summary we present joint PL and photon correlation measurements in small, well isolated InP/InGaP quantum dots that show different trends with increasing temperatures depending on the QD size. In the smaller dot (QD1) the temperature behavior is governed by the hole excitation energy, leading to an increase of both the intensity ratio $I_{X} /\left(I_{X}+I_{\mathrm{XX}}\right)$ and the antibunching time $\tau_{R}$. In the larger dot (QD2) the low-temperature behavior is fixed by the smaller value of the dark-bright exciton splitting, giving rise to a decreasing $\tau_{R}$ with temperature and an essentially temperature independent intensity ratio.

This work has been supported by research contracts of the Spanish Ministry of Education (Grant No. MAT2008-01555/ NAN), Consolider CSD (Grant No. 2006-19), the Community of Madrid (Grant No. CAMS-0505-ESP-0200), and the Spanish Ministry of Science and Innovation (Grant No. Nanoinpho-QD TEC2008-06756-C03-01).
${ }^{1}$ P. Michler, A. Imamoglu, M. D. Mason, P. J. Carson, G. F. Strouse, and S. K. Buratto, Nature (London) 406, 268 (2000).

${ }^{2}$ P. Michler, A. Kiraz, C. Becher, W. V. Shoenfeld, P. M. Petroff, L. Zhang, E. Hu, and A. Imamoglu, Science 290, 2282 (2000).

${ }^{3}$ C. Santori, M. Pelton, G. Solomon, Y. Dale, and Y. Yamamoto, Phys. Rev. Lett. 86, 1502 (2001).

${ }^{4}$ V. Zwiller, H. Blom, P. Jonsson, N. Panev, S. Jeppesen, T. Tsegaye, E. Goobar, M.-E. Pistol, L. Samuelson, and G. Björk, Appl. Phys. Lett. 78, 2476 (2001).

${ }^{5}$ V. Zwiller, T. Aichele, and O. Benson, Phys. Rev. B 69, 165307 (2004); K. Bando and Y. Masumoto, J. Lumin. 128, 855 (2008).

${ }^{6}$ J. D. Cuthbert and D. G. Thomas, Phys. Rev. 154, 763 (1967); P. Palinginis, H. Wang, S. V. Goupalov, D. S. Citrin, M. Dobrowolska, and J. K. Furdyna, Phys. Rev. B 70, 073302 (2004); S. V. Goupalov, R. A. Suris, P. Lavallard, and D. S. Citrin, IEEE J. Sel. Top. Quantum Electron. 8, 1009 (2002).

${ }^{7}$ M. Bayer, G. Ortner, O. Stern, A. Kuther, A. A. Gorbunov, A. Forchel, P. Hawrylak, S. Fafard, K. Hinzer, T. L. Reinecke, S. N. Walck, J. P. Reithmaier, F. Klopf, and F. Schäfer, Phys. Rev. B 65, 195315 (2002).

${ }^{8}$ G. A. Narvaez, G. Bester, and A. Zunger, Phys. Rev. B 72, 245318 (2005).

${ }^{9}$ S. Rodt, A. Schliwa, K. Pötschke, F. Guffarth, and D. Bimberg, Phys. Rev. B 71, 155325 (2005).

${ }^{10}$ J. Persson, T. Aichele, V. Zwiller, L. Samuelson, and O. Benson, Phys. Rev. B 69, 233314 (2004).

${ }^{11}$ G. J. Beirne, M. Reischle, R. Roßbach, W. M. Schulz, M. Jetter, J. Seebeck, P. Gartner, C. Gies, F. Jahnke, and P. Michler, Phys. Rev. B 75, 195302 (2007).

${ }^{12}$ R. Roßbach, M. Reischle, G. J. Beirne, M. Jetter, and P. Michler, Appl. Phys. Lett. 92, 071105 (2008).

${ }^{13}$ W.-M. Schulz, R. Roßbach, M. Reischle, G. J. Beirne, M. Bommer, M. Jetter, and P. Michler, Phys. Rev. B 79, 035329 (2009).

${ }^{14}$ M. Reischle, G. J. Beirne, W.-M. Schulz, M. Eichfelder, R. Roß- bach, M. Jetter, and P. Michler, Opt. Express 16, 12771 (2008).

${ }^{15}$ M. Reischle, G. J. Beirne, R. Roßbach, M. Jetter, and P. Michler, Phys. Rev. Lett. 101, 146402 (2008).

${ }^{16}$ Y. Masumoto, K. Toshiyuki, T. Suzuki, and M. Ikezawa, Phys. Rev. B 77, 115331 (2008).

${ }^{17}$ S. Kako, C. Santori, K. Hoshino, S. Götzinger, Y. Yamamoto, and Y. Arakawa, Nature Mater. 5, 887 (2006).

${ }^{18}$ K. Sebald, P. Michler, T. Passow, D. Hommel, G. Bacher, and A. Forchel, Appl. Phys. Lett. 81, 2920 (2002).

${ }^{19}$ A. Malko, D. Y. Oberli, M. H. Baier, E. Pelucchi, F. Michelini, K. F. Karlsson, M.-A. Dupertuis, and E. Kapon, Phys. Rev. B 72, 195332 (2005).

${ }^{20}$ B. Lounis, H. A. Bechtel, D. Gerion, P. Alivisatos, and W. E. Moerner, Chem. Phys. Lett. 329, 399 (2000); S. Kimura, H. Kumano, M. Endo, I. Suemunei, T. Yokoi, H. Sasakura, S. Adachi, S. Muto, H. Z. Song, S. Hirose, and T. Usuki, Jpn. J. Appl. Phys. 44, L793 (2005).

${ }^{21}$ R. Brouri, A. Beveratos, J.-P. Poizat, and P. Grangier, Opt. Lett. 25, 1294 (2000).

${ }^{22}$ D. W. Snoke, J. Hübner, W. W. Rühle, and M. Zundel, Phys. Rev. B 70, 115329 (2004).

${ }^{23}$ E. Tsitsishvili, R. v. Baltz, and H. Kalt, Phys. Rev. B 67, 205330 (2003).

${ }^{24}$ J. Persson, M. Holm, C. Pryor, D. Hessman, W. Seifert, L. Samuelson, and M. E. Pistol, Phys. Rev. B 67, 035320 (2003).

${ }^{25}$ C. Pryor, M.-E. Pistol, and L. Samuelson, Phys. Rev. B 56, 10404 (1997).

${ }^{26}$ G. A. Narvaez, G. Bester, A. Franceschetti, and A. Zunger, Phys. Rev. B 74, 205422 (2006).

${ }^{27}$ M. Bayer, O. Stern, A. Kuther, and A. Forchel, Phys. Rev. B 61, 7273 (2000).

${ }^{28}$ J. Puls, M. Rabe, H.-J. Wünsche, and F. Henneberger, Phys. Rev. B 60, R16303 (1999). 